$\xi=-$ 国

\title{
Synthesis of Nanocomposite Based on Poly Methylene-imidazole as Effective Catalyst in Oxidation of Some Hydrocarbones
}

\author{
Muhanned Abdul-Redhah Aidan', Wafaa Mahdi Alkoofee ${ }^{2}$, Ala`a A. Sultan³, Wessal M. Khamis ${ }^{4}$, Sinan Medhat ${ }^{5}$ \\ 1,4,5 Mustansiryah University/College of Science /Chemistry department, Baghdad-Iraq \\ ${ }^{2}$ Muthanna University/College of Science/Chemistry Department, Muthanna-Iraq \\ ${ }^{3}$ Dayala University/ College of Science /Chemistry Department, Dayala-Iraq \\ *Corresponding authorE-mail: wessalmetaab@uomustansiriyah.edu.iq
}

\begin{abstract}
The polymers derived from heterocyclic rings like imidazole was prepared and supported to produce catalytic active supported catalyst. This catalyst was characterized using X-ray diffraction (XRD), Fourier-transform infrared spectroscopy (FT-IR), H NMR and UV-visible spectroscopy. The catalyst showed high catalytic activity in the oxidation of cyclohexene and cyclopentene under optimized conditions. In this work cyclohexene and cyclopentene were selected as model alkene for determination the capacity of the prepared imidazole polymer catalyst under optimized conditions of temperature and time of reaction. The catalyst could be readily separated from the catalytic system using uploading 3-5 milligrams of Copper(II), Nickel(II) and Cobalte(II) ions with the surface of polymer the conversion them to nano-particle which are identified by x-ray diffraction. For this research, a statistical method called Response Surface Methodology (RSM) has been used to economize the number of experiments and their meaningful interpretation. The effect of metallated polymer with Copper(II), Nickel(II) and Cobalte(II) were taken to increase the efficiency of oxidation. Optimization results for 0.33 mmole cyclohexene and cyclopentene showed that maximum oxidation efficiency $90 . \%$ was achieved at the optimum conditions: catalyst amount 350 $\mathrm{mg}$, temperature 70.0 , time $3.30 \mathrm{~h}$ and oxidant $=5.25 \mathrm{~m}$ mole.
\end{abstract}

Keywords: Imidazole polymers, nano particles of cobalt(II), copper(II) and nickel(II), oxidation of cyclohexene by nanoparticles.

\section{Introduction}

The interesting of scientists with the using of hetero polymers in the catalysis of organic reactions has been importance field in the recent years. The pyrrole and imidazole polymers have several advantages as catalysts which make them economically and environmentally attractive [Yuxi Liu Guofeng Zhao etal 2015, Yuanhang Ren etal 2015, Marek CYPRYK etal 2013, Pavel Drabina etal2017, Chen,Wei 2006, James P.Collman 2001]. On the other hand, these polymers with their metal complexes have a wide spectrum, approaching the super acid region due to enhancement of oxidation ability for the cyclic alkenes in good yields [Paul Christopher R.Yeisley 2013, G.Hayes etal 2001]. Simple methods of preparation, the possibility of changing the chemical or physical properties or qualities and the work of eight or three nanomaterials of heterogeneous polymers are considered to be the most important characteristics and characteristics of nano-crystalline membrane polymers. The eight and three nano-molecules of heterogeneous polymers were performed in the main reversible roles to form multiple types of fluid through the vacant orbits scattered in the Lewis (II), Ni (II) and Cobalt (II) [Paul J.Chirik 2015, E.Rezaee Nezhad etal 2015, Vladimir Valentinovich Kozlov 2016, Nicola Cioffi,Luisa Torsi etal 2005, Wun Shim etal 2002, Joseph Govan etal 2014]. Considered operations alkenes antioxidants that stimulate polymers pyrrole base factor in the new industrial chemistry, working to stimulate polymers pyrrole, such as olefins and cycled octane basic compounds are good for the process of oxidative stress is working to assess the quality of the potential stimulus in the oxidation of non-non-saturated homogeneous these compounds. Preparation of poly polymers as different as nanoparticles spread on the surface of bentonite as a catalyst and enhancer for epoxides of unsaturated compounds such as alkene[Hossein Salavatia etal 2017]. The nanocomposite that have investigated in corrosion of carbon electrodes have combined effects in oxidation of alkenes quickly [Ezzat Rafiee etal 2017]. The present work deals with the synthesis of nano particles of cobalt, nickel and Copper(II) derived from hetero polymer of methylene-imidazole and their applications as catalyst in oxidation of cyclohexane.

\section{Materials and Methods}

All Chemicals and Solvents materials were provided Sigma Aldrich and BDH chemical companies.

The samples were ground into a fine powder for characterization by: FT-IR spectra were obtained as potassium bromide pellets in the range of $400-4000 \mathrm{~cm}^{-1}$ with a Shimadzu FT-IR spectrometer instrument at Al-Mustansiryah University/College of Science-Chemistry Department. The electronic spectra of the catalyst and their loaded complexes of Nicklate(II), copper(II) and cobalt(II) were recorded on a 160Shimadzu spectrophotometer at Al-Mustansiryah University/College of Science/Chemistry department-physical chemistry research laboratory.

X-ray diffraction (XRD) patterns were recorded with a Philips X-ray diffractometer (Model PW1840) at Baghdad University /Ibn-Haithum, college of education. 
$\mathrm{H}^{1} \mathrm{NMR}$ spectra were recorded on Burker DMX-5000NMR (300- more the broad band around(3400-3396) $\mathrm{cm}^{-1}$ was resulted from the $600 \mathrm{MHz}$ ) spectrophotometer with using $\mathrm{CDCl}_{3}$ as a solvent in Jordan presence of coordinated water molecules in structure of catalysts.

\section{Universities}

Preparation of poly Methylene-Imidazole: The polymer MI was synthesized by beforehand reported techniques, to describe it briefly: 2 $\mathrm{ml}$ of imidazole was dissolved in $10 \mathrm{ml} \mathrm{NaOH}(10 \% \mathrm{w} / \mathrm{v})$ after that $3.25 \mathrm{~g}$ of sodium sulfate was added to above solution. Then, they the mixture stirred for 3.5 hours. After that, a solution containing $3.08 \mathrm{~g}$ of formaldehyde was included into the above solution, keeping the temperature at $25 \mathrm{oC}$ for $30 \mathrm{~min}$. The mixture was centrifuged and the product was washed three times with water and then dried at $80 \mathrm{oC}$ for $3 \mathrm{~h}$ and $60 \mathrm{oC}$ for $2 \mathrm{~h}$ [Tong Liu etal 2016].

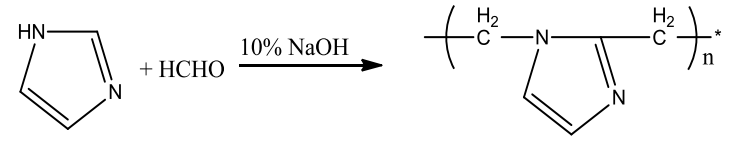

The scheme of MI was shown in scheme 1 .

Scheme1. Synthesis of methylene-imidazole polymer

Preparation of supported catalyst : The polymer metal supported on poly imidazole was synthesized through electrostatic interaction between positive charge of poly methyl imidazole and the anionic dichlorido Cobaltite(II), Nickelite(II) or Copprate(II). The synthesis of supported on poly methyl imidazole was carried out by dissolving copolymer $(1.5 \mathrm{~g})$ in distilled water $(100 \mathrm{ml})$. This solution was added slowly to a synthesized. The mixture was stirred to obtain catalyst. The catalyst was collected using centrifuged and dried under air for $24 \mathrm{hrs}$ at $25 \mathrm{oC}$. The scheme of supported catalyst was shown in scheme2.

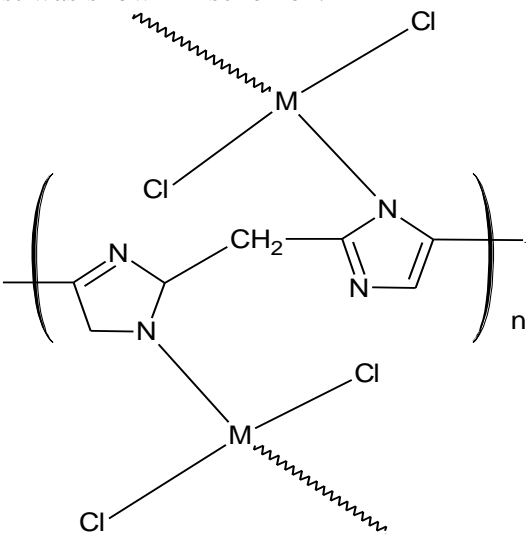

Scheme 2): Proposed scheme for supported catalyst.

\section{Results and Discussions}

Sample characterization: The FT-IR spectra of copolymer showed strong absorptions around 1630,1450 and $2988 \mathrm{~cm}^{-1}$ which are assigning to bonds of $-\mathrm{C}=\mathrm{N},-\mathrm{C}=\mathrm{C}$ and $-\mathrm{C}-\mathrm{H}$ of methylene groups res -pectively, Fig.1. All supported catalyst with $\mathrm{Co}(\mathrm{II}), \mathrm{Ni}(\mathrm{II})$ exhibited medium and broad bands at around (1591$1560) \mathrm{cm}^{-1}$ supporting the linkage of metal ions on the structure of copolymer prepared. The broad absorption around $3387 \mathrm{~cm}^{-1}$ may be assigned to hydrogen bonding in the copolymer chain through $\mathrm{C}=\mathrm{N}$ - of imidazole with the molecules of hydra -ted water assisting in increasing the stability of the imidazole-methylene copolymer. The $-\mathrm{C}-\mathrm{H}$ bending of imidazole ring was shown at $852 \mathrm{~cm}^{-1}$ as weak band whereas the $-\mathrm{CH}_{2}$ - moiety was observed at 2859 and $711 \mathrm{~cm}^{-1}$ associated with vibration and rocking motions in IR spectra[Guillermo Mendoza-Díaz 2002, Qingzhong Hu 2008, Yuichi Ikuta2003, Augusto Rivera 2009, Tarun Kumar Misra 1998]. The comparison of IR spectra of imidazole copolymer with IR spectra of its nickel(II) and cobalt(II) catalysts, Figures(2,3)displays new bands in the regions (400-600) $\mathrm{cm}^{-1}$ assigning to $\mathrm{M}-\mathrm{N}$ bonds. As well as the shift in the vibrations of $-\mathrm{C}=\mathrm{N}-$ and-C $=\mathrm{C}$ - functional groups to $1631 \mathrm{~cm}^{-1}$ confirm the formation of metal catalyst [Lionel E etal 2006, A.Mašlejováa 2006, Yao-Yu etal 1999, R.Carballoa 2004, M.FIskander etal 2000, Ikuko Katsuki etal 2002]. Further-

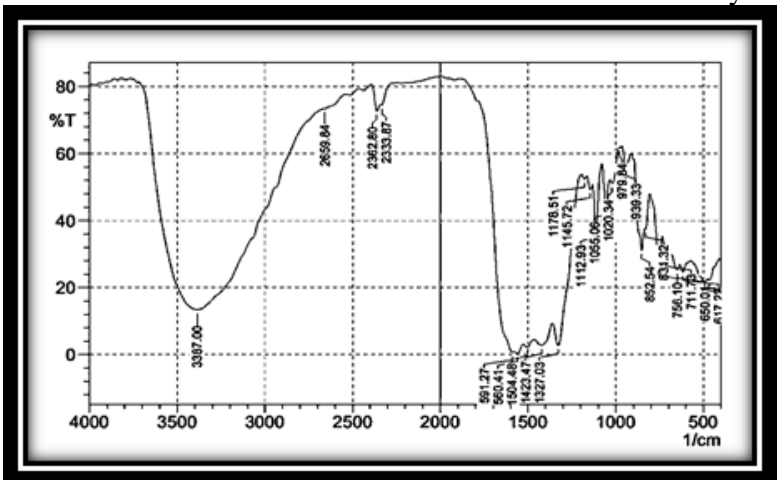

Fig. 1: FT-IR spectra of imidazole-methylene copolymer

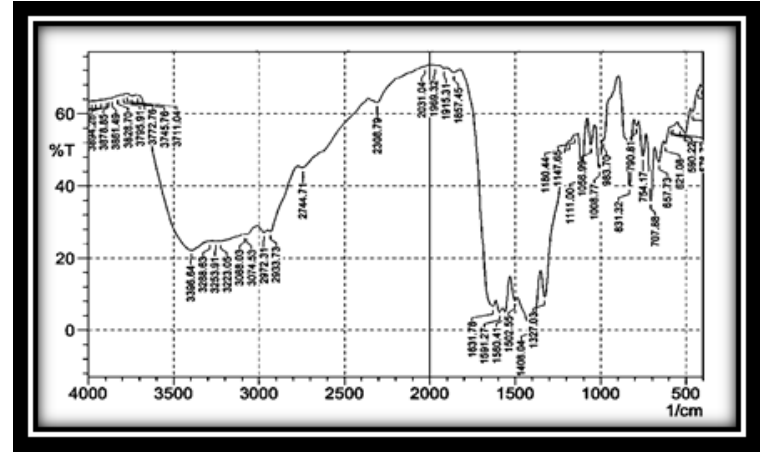

Fig. 2: FT-IR spectra of cupper(II)-Imidazole catalyst.

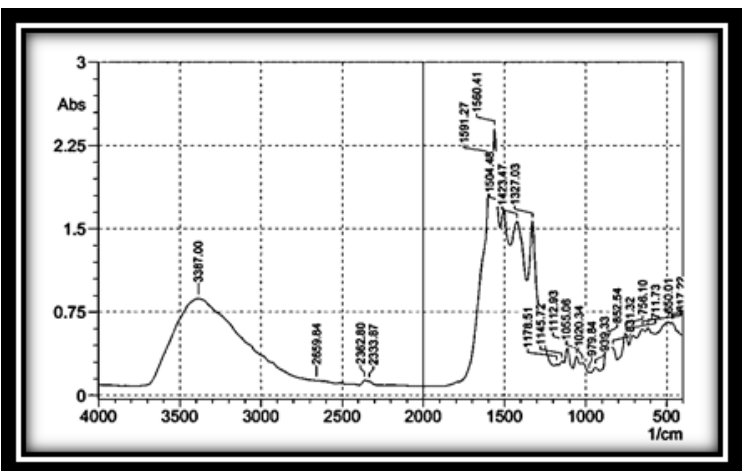

Fig. 3: FT-IR spectra of cobalt(II)-Imidazole catalyst.

NMR characterization:The $\mathrm{H}^{1} \mathrm{NMR}$ spectra of imidazole copolymer in $\mathrm{CDCl} 3$ showed resonance of aromatic $-\mathrm{C}-\mathrm{H}$ in the imidazole ring around 7-8ppm. As well as the multiple peaks at 2$4.5 \mathrm{ppm}$ may be assigned to aliphatic $\mathrm{CH}_{2}$ - moiety of methylene group as shown at fig.(4). Up on formation the catalyst with Cobalt and Nickel the changes are distinct in $\mathrm{H}^{1} \mathrm{NMR}$ of Cobalt(II) and Nickel(II)catalysts. The $\mathrm{H}^{1} \mathrm{NMR}$ spectra of cobalt(II) formed with imidazole copolymer,figure(5) exhibits no clear peaks due to the odd electrons in the $3 \mathrm{~d}^{7}$ configuration causing a little support for the long chain of the metal catalyst[Guoli Huang etal 2011, Umasankar Ray etal 2004, Markus Andersson etal 2010, Giovanni Tabbì etal 1997, Markus Albrecht etal 1999, Scott J.Moore etal 1999, Ying-Ying Liu etal 2007, Ronald Dean But 1958]. 


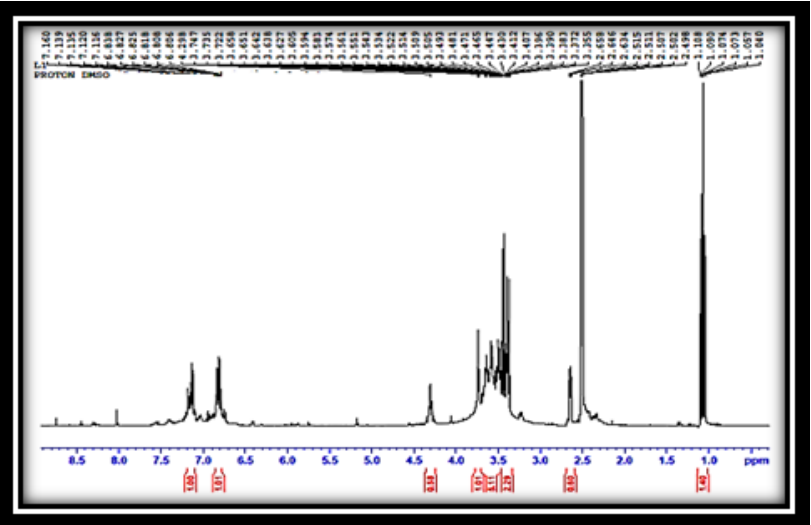

Fig.4: $\mathrm{H}^{1} \mathrm{NMR}$ of methylene-imidazole in $\mathrm{CDCl}_{3}$.

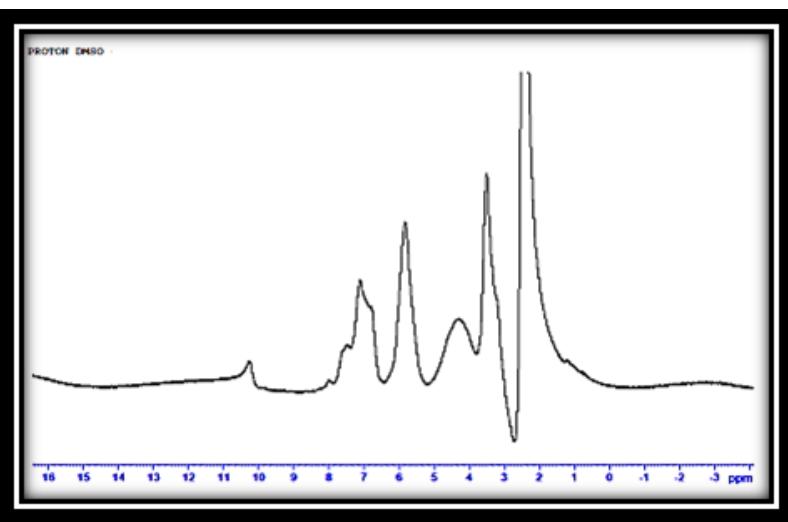

Fig 5: $\mathrm{H}^{1} \mathrm{NMR}$ spectra of Cobalt (II)- imidazole-methylene polymer in $\mathrm{CDCl}_{3}$

UV-Visible spectra of the imidazole-methylene copolymer and it's Cobalt(II), Nickel(II) and Copprate (II) catalysts : The UVVisible spectra of imidazole copolymer in $\mathrm{CH}_{3} \mathrm{CN}$ and $\mathrm{CH}_{2} \mathrm{Cl}_{2}$ displayed weak intensity bands around (210-245) nm associated to the $\pi \rightarrow \pi *$ transitions of $-\mathrm{C}=\mathrm{C}$ - and $-\mathrm{C}=\mathrm{N}$ - functional groups. As well as the $\mathrm{n} \rightarrow \pi^{*}$ transitions of the chromophores moiety was observed in the range (350)nm [Mahendra Kumar Trivedi etal 2015, Bao Shan Huang etal 1979, Mariel M.Muir etal 1988, S. A. Cotton 2004], Fig.(6) clarified the main bands of imidazole copolymer. The main bands of copolymer metal complexes were shifted to lower or higher vibrations compared to the original spectrum of copolymer. These referred to coordinate of metal to active functional group of imidazole copolymer.

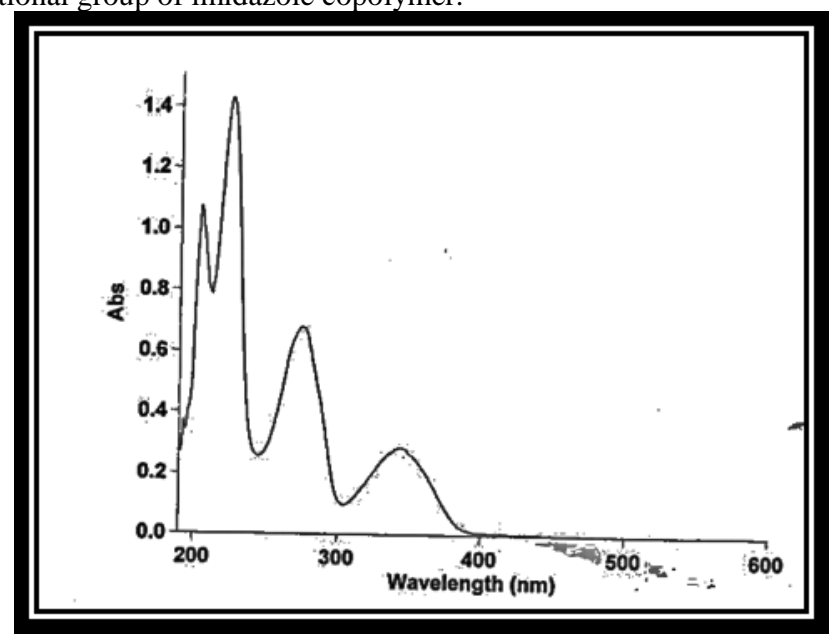

Fig. 6: UV of copolymer imidazole

X-Ray diffraction of Co (II) and Ni (II) catalysts of imidazole copolymers:

XRD patterns of imidazole copolymer indicated that the transition elements under study and derived from their hydrated metal chlorides,i.e, $\mathrm{CoCl}_{2} \cdot 6 \mathrm{H}_{2} \mathrm{O}$ and $\mathrm{NiCl}_{2} \cdot 6 \mathrm{H}_{2} \mathrm{O}$ have remarkably immobilized on the surface position of heterocyclic ring through the aggregation of copolymer chain via nitrogen atom with empty orbitals of Lewis acids of metal ions[41-43]. Moreover the advanced diagnosis of peaks of imidazole-methyl copolymer with Cobalt(II) ion (Fig. 7a) investigated that heterocyclic moiety was strongly prepared in the proposed structure. The powder XRD patterns $(0<\Theta<65)$ of poly-imidazole obtained from the samples are shown in Figure (3b) and exhibited amorphous structure of the catalysts. By the same manner the x-ray diffraction curves for Nickel(II) catalysts recorded a range of angle diffraction around $0<\Theta<65$ and $0<\Theta<78$ indicating the amorphous state of the two catalysts[S. Ganeshraja etal 2014, M. Bouchouit etal 2016, G. -H. Cui etal 2012, Norah Barba-Behrensetal 1991, Shui-Sheng Chen etal 2012].

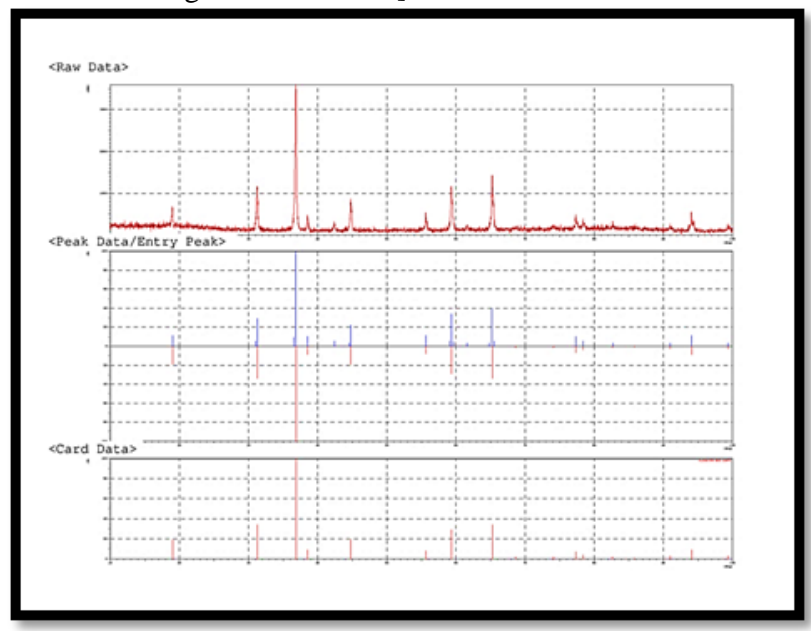

Fig.8: X-Ray diffraction of Cobalt - copolymer

\section{Conclusion}

From Our study, it was concluded that he efficiency of the catalyst which was prepared by doped of an active catalyst at polymers derived from heterocyclic compound like imidazole ring.

Preparation of catalyst was prepared as nano particles of metals ions which deposited at polymer surface.

Imidazole polymer doped with $\mathrm{Co}(\mathrm{II})$ and $\mathrm{Ni}(\mathrm{II})$ metals as catalyst complexes used to catalyze the oxidation of unsaturated compounds.

Polymers - imidazole-metals complexes showed good efficiency at oxidation catalyzed of cyclohexanes and cyclopentene.

\section{References}

[1] Yuxi Liu Guofeng Zhao Dingsheng Wang Yadong Li. 2015. Heterogeneous catalysis for green chemistry based on nanocrystals. National Science Review, 2(2):150-166.

[2] Yuanhang Ren,Meiyin Wang,Xueying Chen,Bin Yue\&Heyong He. 2015. Heterogeneous Cat -alysis of Polyoxometalate Based Organic-Inorganic Hybrids. Materials, 8:1545-1567.

[3] Marek Cypryk, Piotr Pospiech. 2013. Polysiloxanes as supports for catalysts. Centre of Molecular and Macromolecular Studies, Polish Academy of Sciences, Łódź, Poland) Chemik,67,12:1173-1180,.

[4] Pavel Drabina,Jan Svoboda \& Miloš Sedlák. 2017. Recent Advances in $\mathrm{C}-\mathrm{C}$ and $\mathrm{C}-\mathrm{N}$ Bond Forming Reactions Catalysed by Polystyrene-Supported Copper Complexes. Molecules , 22, 865.

[5] Chen,Wei. 2006. Studies on immobilized polymer-bound imidazole copper(II) complexes as catalysts. Univ. medical center Groningen, University of Groningen.

[6] James P.Collman,Min Zhong,Chi Zhang \& Simona Costanzo. 2001 Catalytic Activities of $\mathrm{Cu}(\mathrm{II})$ Complexes with Nitrogen-Chelating Bidentate Ligands in the Coupling of Imidazoles with Arylboronic Acids.J.Org.Chem.,66(23):7892-7897 . 
[7] Christopher R.Yeisley. 2013. Synthesis of Early Transition Metal Complexes Supported by Pyrrolyl and Indolyl Based Ligands 'Thesis, The University of Toledo,.

[8] Paul G.Hayes,Warren E.Piers,Lawrence W.M.Lee,Lisa K. Knight, Masood Parvez, Mark R.J.Elsegood \&William Cleg. 2001. Dialkylscandium Complexes Supported by â-Diketiminato Ligands: Synthesis, Characterization and Thermal Stability of a New Family of Organoscandium Complexes. Organometallics, 20:2533-2544.

[9] Paul J.Chirik. 2015. Iron- and Cobalt-Catalyzed Alkene Hydrogenation: Catalysis with Both Redox-Active and Strong Field Ligands. cc.Chem.Res.,48 (6):1687-1695.

[10] E.Rezaee Nezhad,S.Karimian \& S.Sajjadifar. 2015. Imidazole Functionalized Magnetic Fe3O4 Nanoparticles a Highly Efficient and Reusable Brønsted Acid Catalyst for the Regiosele -ctive Thiocyanation of Aromatic and Heteroaromatic Compounds at Room Temperature in Water:Ethanol. J. of Sci., Islamic Republic of Iran, 26(3):233-240.

[11] Vladimir Valentinovich Kozlov. 2016. Synthesis of Nanomaterials and Hetero structures. National University of Science and Technology.

[12] Nicola Cioffi,Luisa Torsi,Nicoletta Ditaranto, Giuseppina Tantillo, Lina Ghibelli, Luigia Sabbatini, Teresa Bleve-Zacheo,Maria D'Alessio, P.Giorgio Zambonin \&Enrico Traversa. 2005. Copper Nanoparticle/Polymer Composites with Antifungal and Bacteriostatic Properties Chem.Mater.17 (21): 5255-5262.

[13] Wun Shim,Won-Tae Noh,Jiwoon Kwon,Jung Young Cho,KyungSoo Kim\&Dong Hee Kang “Preparation of Copper Nanoparticles in Cellulose Acetate Polymer and the React -ion Chemistry of Copper Complexes in the Polymer. Bull.Korean Chem.Soc.,23(4):563.

[14] Joseph Govan\&Yurii K.Gun'ko. 2014. Recent Advances in the Application of Magnetic Nano -particles as a Support for Homogeneous Catalysts“ Nanomaterials, 4:222-241.

[15] Hossein Salavatia,Abbas Teimouria,Shahnaz Kazemi. 2017. Synthesis and characterization of nanocomposite based on polymer as a highly effective supported catalyst in oxidation of alkenes. Asian Journal of Green Chemistry,98-115.

[16] Ezzat Rafiee,Ayoob Shahbazirad\&Maryam Khodayari. 2017. Preparation and characterizea -tion of nanocomposite of graphitic carbon nitride and $\mathrm{TiO} 2$ as a porous support for nano catalyst for desulfurization process. J.Saud.Chem. Soc., 21(8):943-953.

[17] Tong Liu,Haiyang Cheng,Weiwei Lin,Chao Zhang,Yancun Yu\&Fengyu Zhao. 2016. Aerobic Catalytic Oxidation of Cyclohexene over TiZrCo Catalysts“Catalysts, 6(2):24.

[18] Guillermo Mendoza-Díaz, Willem,L. Driessen, Jan Reedijk, SybGorter, Laura Gasque, Kimberly R.Thompson. 2002. Synthesis, characterization and $\mathrm{X}$-ray structures of the new dinucleating ligand 2,8-dimethyl-1,4,5,6,7,10,11,12-octahydroimidazo[4,5-h]imidazo [4,5-c] [1,6]diazecine-5,11-diethanoic acid and its $\mathrm{Cu}$ (II) complex; an alternating chain of $\mathrm{Cu}(\mathrm{II})$ ions, coupled both intramolecularly and intermolecularly. Inorganica Chimica Acta,Vol. 339:51-59.

[19] Qingzhong Hu, Matthias Negri, Kerstin Jahn-Hoffmann, Yan Zhuang, Sureyya Olgen, Marc Bartels, Ursula MüllerVieira,ThomasLauterbach and Rolf W. Hartmann. 2008. Synthesis, biological evaluation, and molecular modeling studies of methylene imidazole substituted biaryls as inhibitors of human $17 \alpha$ hydroxylase-17,20-lyase (CYP17)-Part II: Core rigidification and influence of substituents at the methylene bridge. Bioorganic \& Medicinal Chemistry, 16(16): 7715-7727.

[20] Yuichi Ikuta,Makoto Ooidemizu,Yuichi Yamahata, Masahiro Yamada,Shutaro Osa, Naohide Matsumoto, Seiichiro Iijima,.Masaaki Kojima , Françoise Dahan and Jean-Pierre Tuchagues. 2003. A New Family of Spin Crossover Complexes with a Tripod Ligand Containing Three Imidazoles: Synthesis, Characterization and Magnetic Properties FeIIH3LMe](NO3)2 $1.5 \mathrm{H} 2 \mathrm{O}$,[FeIIILMe]·3.5H2O,

[FeIIH3LMe][FeIILMe]NO3 and [FeII H3LMe] [FeIIILMe] (NO3)2 (H3LMe=Tris[2-((2-methyl imidazol-4-yl) methylidene) amino) ethyl] amine) .Inorg. Chem. 42, 22:7001-7017.

[21] Augusto Rivera,Diego Quiroga,Jaime Ríos-Motta, Juan Carda \&Gabriel Peris. 2009. Synthesis, Characterization and X-ray Crystal Structure of the Di-Mannich Base2,2'-(3aR,7aR/ 3aS,7aS)Hexahydro-1H-benzo[d]imidazole-1,3(2H)-diyl) bis (methylene)bis(4-methyl-phenol). Journal of Chemical Crystallography, 39(11):827-830.

[22] Tarun Kumar Misra,Debasis Das,Chittaranjan Sinha \& Chandan Kumar Pal. 1998. Chemistry of Azoimidazoles: Synthesis, Spectral Characteri -zation, Electrochemical Studies and X-ray Crystal
Structures of Isomeric Dichloro Bis[1-alkyl-2-(arylazo) imidazole] Complexes of Ruthenium(II). Inorg. Chem. 37, 8:1672-1678.

[23] Lionel E.Cheruzel,Matthew R.Cecil,Sara E.Edison, Mark S.Mashuta,Michael J.Baldwin \&Robert M.Buchanan. 2006. Structural and Spectroscopic Characterization of Copper(II) Complexes of a New Bisamide Functionalized Imidazole Tripod and Evidence for the Formation of a Mononuclear End-On $\mathrm{Cu}-\mathrm{OOH}$ Species. Inorg.Chem.,45 (8) :3191-3202.

[24] A.MašlejováaR.IvanikováaI.SvobodabB.PapánkováaL.DlháňaD.Mi klošaH.FuessbR.Bočaa. 2006. Structural characterization and magnetic properties of hexakis (imidazole) nickel (II)bis(formate), bis (chloroacetate), bis(2-chloropropionate) and hexakis(1-methylimidazole)nickel(II)chloride dehydrate. Polyhedron,25(8):18231830.

[25] Yao-YuWangaQianShiaQi-ZhenShiaYi-CiGaobZhongYuanZhou 'Syntheses, character -rization and crystal structure of copper(II) $\alpha, \beta$-unsaturated carboxylate complexes with imidazole. 1999. Polyhedron, 18 (15):2009-2015.

[26] R.CarballoaA.CastiñeirasbB.CoveloaE.GarcíaMartínezaJ.NiclóscE.M.Vázquez-Lópeza. 2004 . Solid state coordination chemistry of mononuclear mixed-ligand complexes of $\mathrm{Ni}(\mathrm{II})$, $\mathrm{Cu}$ (II) and $\mathrm{Zn}$ (II) with $\alpha$-hydroxycarboxylic acids and imidazole. Polyhedron,23(9):1505-1518.

[27] M.FIskander,T.EKhalil,RWerner,WHaase,ISvobod\&HFuessc. 2000 Synthesis, characterization and magnet -ochemical studies of some imidazole and imidazolate copper(II) ternary complexes derived from $\mathrm{N}$-salicylidenearoylhydrazines. X-ray crystal and molecular structures of [(N-salicylidene-p-chlorobenzoylhydrazine $)$ $\mathrm{ONO}(-2)$ imidazole $]$ Copper(II) monohydrate and dimeric $[(\mathrm{N}$ salicylid -ene-p-methyl benzoylhydrazine) $\mathrm{ONO}(-2)$ imida -zole ] copper(II) hemihydrate. Polyhedron, 19 (10): 1181-1191.

[28] S.Materazzi,S.Vecchio,L.W.Wo\&cS.De Angelis Curtis. 2012.TGMS and TG-FTIR studies of imidazole-substituted coordination compounds: $\mathrm{Co}(\mathrm{II})$ and $\mathrm{Ni}(\mathrm{II})$-complexes of bis(1-methylimidazol2-yl) ketone. Thermochimica Acta,543:183-187.

[29] Julie Brown ,Ian Hamerton and Brendan J. Howlin. 2000. Preparation, characterization, and thermal properties of controllable metalimidazole complex curing agents for epoxy resins.J. of Appl. Poly. Sci.,75(2):201-217.

[30] Ikuko Katsuki, Yuri Motoda,Yukinari Sunatsuki, Naohide Matsumoto, Toshio Nakashima \& Masaaki Kojima. 2002. Spontaneous Resolution Induced by Self-Organization of Chiral SelfComplementary Cobalt(III) Complexes with Achiral Tripod-Type Ligands Containing Three Imidazole Groups. J.Am.Chem. Soc., 124 (4):629-640.

[31] Guoli Huang,Hongsheng Sun,Xiaoji Qiu,Yingzhong Shen,Juli Jiang\&Leyong Wang. 2011. Synthesis, structural characterization of benzimidazole-functionalized $\mathrm{Ni}$ (II)and $\mathrm{Hg}$ (II) N-heterocyclic carbene complexes and their applications as efficient catalysts for Friedel-Crafts alkylations. Journal of Organometallic Chemistry, 696 (18) :2949-2957.

[32] Umasankar Ray,Debasis Banerjee,Golam Mostafa,Tian-Huey Luc\&Chittaranjan Sinha. 2004. Copper coord -ination compounds of chelating imidazole-azo-aryl ligand.The molecular structures of bis[1-ethyl-2-(p-tolylazo) imidazole]-bis-(azido) copper(II) and bis[1-methyl-2-(phenylazo)imidazole]-bis (thiocyanato) Copper(II). New J.Chem.,(12).

[33] Markus Andersson, Jesper Hedin, Patrik Johansson , Jonas Nordström and Magnus Nydén. 2010. Coordination of Imidazoles by $\mathrm{Cu}$ (II) and $\mathrm{Zn}$ (II) as Studied by NMR Relaxometry, EPR, farFTIR Vibrational Spectroscopy and Ab Initio Calculations: Effect of Methyl Substitution “J. Phys. Chem. A, 114 (50) :13146-13153.

[34] Giovanni Tabbì, Willem L. Driessen, Jan Reedijk , Raffaele P. Bonomo, Nora Veldman and Anthony L. Spek. 1997. High Superoxide Dismutase Activity of a Novel,Intramolecularly ImidazolatoBridged Asymmetric Dicopper(II) Species. Design, Synthesis, Structure, and Magnetism of Copper(II) Complexes with a Mixed Pyrazole-Imidazole Donor Set. Inorg. Chem., 36 (6):1168-1175.

[35] Markus Albrecht,Klaus Hübler,Thomas Scheiring \&Wolfgang Kaim. 1999. $\mathrm{Cu}$ (I)and $\mathrm{Cu}$ (II) complexes of the bidentate imidazole/thioether ligand 1-methyl-2-(methyl thiomethyl)-1H-benzim idazole. Inorganica Chimica Acta, 287(2):204-208.

[36] Scott J.Moore,Alexander Kutikov,Rene J.Lachicotte \& Luigi G.Marzilli.1999. Methyl B12 Models Containing Unsubstituted Imidazole As an Axial Ligand Investigated by Structural and NMR Spectroscopic Methods. Evidence that $\mu$-Imidazolato-Bridged Dimers Are Formed by Base Addition to Some Analogues with Mac- 
rocyclic Equatorial Ligands Incorporating BF2.Inorg. Chem., 38(4):768-776

[37] Ying-Ying Liu,Jian-Fang Ma,Jin Yang\&Zhong-Min Su. 2007. Synthesis and Characterization of Six Coordination Polymers of $\mathrm{Zn}$ (II)and $\mathrm{Co}(\mathrm{II})$ with 1,3,5-Benzenetricarboxylate Anion and Bis (imidazole) Ligands. Inorg.Chem.,46(8):3027-3037.

[38] Ronald Dean But. 1958. The Ultraviolet Absorption Spectra Of Histamine, Histadene, and imidazole; Effect of $\mathrm{pH}$ and Certain foreign ions on the spectrum of Histamine. Master Thesis , Kansas State University, Manhattan, Kansas.

[39] Mahendra Kumar Trivedi1 , Alice Branton1, Dahryn Trivedi1, Gopal Nayak1 , Gunin Saikia and Snehasis Jana. 2015. Physical and Structural Characterization of Biofield Treated Imidazole Derivatives. Nat. Prod. Chem. Res., 3:5.

[40] Bao Shan Huang, Mary Jane Lauzon And James C. Parham. 1979. Synthesis and properties of alkylated imidazoles.16(4): 811-813.

[41] Mariel M.MuirMayra E.Cadiz1AdrianaBaez. 1988. Synthesis and characterization of new platinum(II) complexes containing thiazole and imidazole donors . Inorganica Chimica Acta,151(3):209-213.

[42] S. A. Cotton. 2004. Annual Reports Section "A" (Inorganic Chemistry).Iron, cobalt and nickel chapter, Royal Society of Chemistry, 100 .

[43] Ganeshraja,K.Rajkumar,S.Thirumurugan,K.Anbalagan. 2014. Synthesis, Characterization, Structural, Electrochemical and Magnetic Studies of Monomeric [NiII(1-MeIm)6]C12.H2O Complex. J. Environ. Nanotechnol.3(3):121-128.

[44] M. Bouchouit,A. Bouraiou,S. Bouacida,A. Belfaitah and H. Merazig. 2016. Structural characterization of a new cobalt(II)complex of 1-benzyl-5-methyl-1H-imidazole.Journal of Structural Chemistry, 57(4): 835-839.

[45] G. H. Cui,J.,C.Geng,T.,F.Liu,X. Peng,C.,H.He and C., H. Jiao. 2012. Structure and characterization of a 1d double chain copper(II) complex $\{[\mathrm{Cu}(\mathrm{BIX}) 2(\mathrm{H} 2 \mathrm{O}) 2](\mathrm{PhCOO}) 2\}$ n. Journal of Structural Chemistry, 53(3):509-513.

[46] Norah Barba-Behrens Ana María Mutio-Rico Pedro Joseph-Nathan Rosalinda Contreras. 1991. Preparation and characterization of new transition metal complexes of nitroimidazoles. X-Ray crystal structures of two copper complexes: bis-[( $\mu$-chloro $)$ chloro-bis-(1-(2hydroxyethyl)-2-methyl-5-nitroimi -dazole) copper(II)] and dichloro-bis-(2-methyl-s-nitroimidazole) Copper(II). First observation of nitro group coordination to the metal ion in these heterocycles. Polyhedron, 10(12):1333-1341.

[47] Shui-Sheng Chen, Zhi-Hao Chen, Jian Fan, Taka-aki Okamura, Zheng-Shuai Bai, Mei-Fang Lv and Wei-Yin Sun. 2012. Synthesis and Characterization of Metal Complexes with Mixed 4Imidazole-Containing Tripodal Ligand and Varied Dicarbox -ylic Acid.Cryst. Growth Des., 12 (5):2315-2326. 